\section{O Cenário da Pós-Graduação em Educação Física: Contextos e possibilidades na região sul do Brasil}

\author{
The reality of graduate courses in Physical \\ Education: Context and possibilities in \\ Southern Brazil
}

Helder Madruga de Quadros ${ }^{1}$

Mariângela da Rosa Afonso ${ }^{2}$

José Antonio Bicca Ribeiro ${ }^{3}$

\section{Resumo}

As mudanças ocorridas no cenário da Pós-Graduação brasileira promoveram um crescimento na quantidade de programas, inclusive na área da Educação Física. No entanto, uma constante avaliação e reflexão sobre esse crescimento é necessária. Desse modo, o objetivo do estudo é sinalizar a partir de dados descritivos o cenário da Pós-Graduação em Educação Física no Brasil, com ênfase na região Sul. O estudo foi realizado a partir de uma busca documental nos relatórios da avaliação da CAPES, do triênio 2009/20011, onde foram coletadas as informações referentes: a) caracterização dos programas, b) estrutura institucional e c) avaliação. Ao buscar mapear as estruturas de construção de cada um dos programas de Pós-Graduação e suas linhas de investigação científica, percebemos que na região Sul, das 28 linhas de pesquisa que compõem os programas de Pós-Graduação, 17 são relacionadas à área das Ciências Biológicas/ Saúde, comprovando a superioridade. Quando analisamos os programas de Pós-Graduação por região e conceitos atribuídos, a região Sudeste apresentam os programas melhores avaliados e os cursos de doutorado mais consolidados. Consideramos que o momento vivido na PósGraduação tem gerado tensões tanto no que diz respeito ao trabalho docente quanto às áreas de produção do conhecimento. Por outro lado, é possível que a partir de um reordenamento e aproximação das áreas e linhas de pesquisa, possa haver um fortalecimento e incremento de novas linhas investigativas na área.

\section{Palavras-chave}

Pós-Graduação; Educação Física; Avaliação Educacional.

\begin{abstract}
The changes in the scene of Post-graduate Brazilian promoted a growth of programs, including in the area of Physical Education, however, some discussions are still needed. In this way, the aim of the study is to signal the descriptive data from the scene of the Postgraduate in Physical Education in Brazil, with emphasis on the Southern region. The study was conducted from a documentary search in the reports from CAPES and CNPq in the year 2012. For the collection of data we used an analysis model divided into three areas: a) characterization of the programs, b) institutional structure and c) evaluation. By seeking to map building structures of each of the programs Postgraduate and its lines of scientific research, we realized that the region south of 28 research lines that make up the Postgraduate programs, 17 are related the area of Sciences Biological/Health, proving the superiority. When we analyze programs Postgraduate by region and attributed concepts, the Southeast have the best programs evaluated and doctoral courses more consolidated. We believe that the moment lived in Post-graduate, has generated tensions both as regards to teaching as to areas of knowledge production. Other hand, it is possible that from a reordering and approach among areas and lines of research, there can be a increment and strength of new investigative lines in the area.
\end{abstract}

\section{Keywords}

Postgraduate; Physical Education; Educational Measurement.
Rev Bras Ativ Fis Saúde p. 576-584 DOI:

http://dx.doi.org/10.12820/rbafs.v.18n5p576

1 Programa de Pós-Graduação em Educação - Universidade Federal de Santa Catarina (UFSC)

2 Programa de Pós-Graduação em Educação Física - Universidade Federal de Pelotas (UFPEL)

3 Programa de Pós-Graduação em Educação Física - Universidade Federal de Pelotas (UFPEL). Bolsista CAPES. 


\section{INTRODUÇÃO}

Consideramos que as atividades de pesquisa, no contexto da Pós-Graduação, dentro das universidades, respondem por grande parte das investigações científicas do país, constituindo um dos vetores que permite a passagem da universidade da condição de apenas transmissora para também produtora do conhecimento. As características fundamentais dos Programas de Pós-Graduação no Brasil, com seus propósitos mais específicos, só passaram a ser definidas ao final da década de sessenta, momento de aceleração do crescimento econômico e significativo apoio financeiro de verbas públicas ${ }^{1}$.

Franco ${ }^{2}$, buscando traçar uma trajetória, diz que a década de 60 foi a de promulgação de políticas estabelecedoras de condições básicas; a década de 70 foi de criação e expansão de cursos, a dos planos globais articuladores; a década de 80 se apresentou como de consolidação, já a década de 90, destaca-se como um momento de crítica, mas ao mesmo tempo de reestruturação.

Para a autora, a década de 90 teve, como traço diretivo, a busca de novas articulações em decorrência, não só da limitação de recursos que incide sobre todas as áreas, mas, também, de tendências mais amplas marcadas pela internacionalização da economia. Os marcos regulatórios, como os Planos Nacionais da Pós-Graduação apontam para um crescimento equânime do sistema nacional de Pós-Graduação, visando o desenvolvimento científico, tecnológico, econômico e social do país, por meio de seis principais diretrizes: estabilidade e indução, estratégias para melhoria do sistema, financiamento e sustentabilidade, novos modelos, políticas de cooperação internacional e de formação de recursos humanos no exterior e, avaliação e qualidade.

Nas diretrizes atuais, contidas no Plano Nacional de Pós-Graduação (20112020) estão demarcadas as futuras políticas que devem fomentar o desenvolvimento nacional deste sistema. O Plano estabelece como diretrizes para o desenvolvimento da Pós-Graduação: a) a expansão do Sistema Nacional de Pós-Graduação (SNPG), a primazia da qualidade, a quebra da endogenia e a atenção à redução das assimetrias; b) a criação de uma nova agenda nacional de pesquisa e sua associação com a pós-graduação; c) o aperfeiçoamento da avaliação e sua expansão para outros segmentos do sistema de ciência, tecnologia e inovação; d) a multidisciplinaridade e a interdisciplinaridade entre as principais características da pós-graduação e importantes temas da pesquisa; e) o apoio à educação básica e a outros níveis e modalidades de ensino, especialmente o ensino médio ${ }^{3}$.

No campo da Educação Física percebe-se uma série de discussões a respeito da configuração do campo relacionado à produção de conhecimento e avanços na PósGraduação, onde parece haver uma diferença entre as áreas, devido a pluralidade relacionada tanto ao corpo docente (área de formação), quanto na produção científica ${ }^{4-6}$.

Desse modo, o objetivo do estudo é sinalizar a partir de dados descritivos o cenário da Pós-Graduação em Educação Física no Brasil, com ênfase na região Sul, considerando que a partir do aporte teórico construído, fossem elucidadas as transformações vividas na área.

\section{MÉTODO}

O presente estudo insere-se no âmbito da abordagem qualitativa possuindo um delineamento descritivo, onde, utilizaram-se as fontes documentais para a sua construção ${ }^{7}$. A coleta de dados foi realizada, no ano de 2012, a partir dos relatórios 
de avaliação disponibilizados nos sites da Coordenação de Aperfeiçoamento de Pessoal de Nível Superior (CAPES) onde foram coletadas informações sobre a configuração dos programas (linhas de pesquisa e áreas de concentração), resultados da avaliação trienal e corpo docente, possibilitando a eleição dos elementos que sinalizam o crescimento dos programas e áreas na Pós-Graduação brasileira como também na área específica da Educação Física.

Foi organizada uma planilha com as informações referentes aos programas, construída tendo como base a figura 1. A partir da análise, a caracterização dos programas foi desenvolvida com base nos documentos institucionais, onde foram criados três eixos. O primeiro deles representa a caracterização dos programas, o segundo, a sua estrutura institucional, e o último eixo, refere-se aos resultados da avaliação institucional.

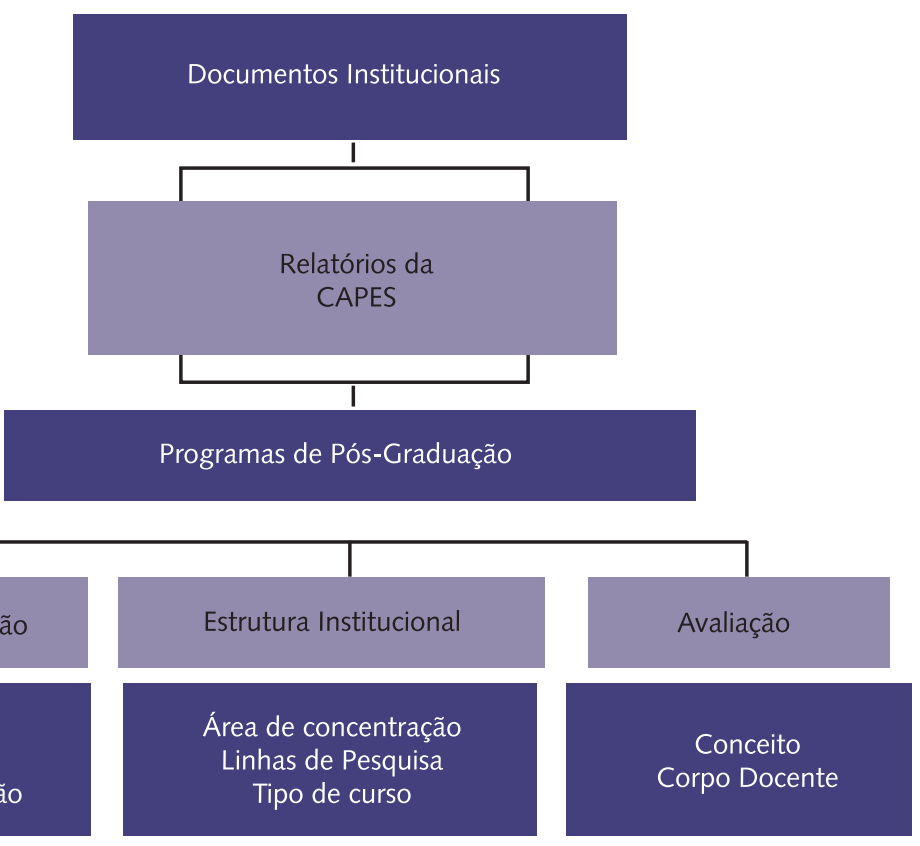

Figura 1 - Organograma da coleta de dados.

\section{RESULTADOS}

Os resultados sinalizaram que nos últimos anos aconteceu aumento significativo relacionado ao número de cursos de Pós-Graduação, bem como, a qualificação das pesquisas na área. O sistema avaliativo da Pós-Graduação tem provocado reformas nos programas fomentando a qualidade e expansão dos cursos.

A fim de aproximar o contexto da Pós-Graduação no Brasil com as estratégias surgidas na área da Educação Física, apresentamos o panorama atual da Pós-Graduação na área da Educação Física no Brasil. No quadro 1, estão descritos os programas de mestrado e doutorado do Brasil segundo o conceito de cada um de acordo com a última avaliação trienal da CAPES. Ao analisarmos o quadro, podemos perceber que os cursos de mestrado relacionados à área da Educação Física, estão consolidados, possuindo um conceito atribuído, porém, existe ainda uma diferenciação quanto às notas atribuídas, prevalecendo na região Sudeste os maiores conceitos. Há também na região Sudeste uma concentração dos programas de doutorado. 
Quadro 1 - Programas de Pós-Graduação na área de Educação Física no Brasil.

\begin{tabular}{|c|c|c|c|c|c|}
\hline & \multirow{2}{*}{ Estado } & \multirow{2}{*}{ Universidade } & \multirow{2}{*}{ Programa } & \multicolumn{2}{|c|}{ Conceito } \\
\hline & & & & Mestrado & Doutorado \\
\hline \multirow{13}{*}{ SUDESTE } & \multirow{3}{*}{ RJ } & Universidade Salgado de Oliveira (UNIVERSO) & Ciências da Atividade Física & 3 & - \\
\hline & & Universidade Federal do Rio de Janeiro (UFRJ) & Educação Física & 3 & - \\
\hline & & Universidade Gama Filho (UGF) & Educação Física & 4 & 4 \\
\hline & \multirow{6}{*}{ SP } & Universidade Estadual Paulista (UNESP/RC) & Ciências da Motricidade & 6 & 6 \\
\hline & & Universidade Cruzeiro do Sul (UNICSUL) & $\begin{array}{l}\text { Ciências do Movimento } \\
\text { Humano }\end{array}$ & 4 & 4 \\
\hline & & Universidade de São Paulo (USP) & Educação Física & 6 & 6 \\
\hline & & Universidade de Campinas (UNICAMP) & Educação Física & 4 & 4 \\
\hline & & Universidade Metodista de Piracicaba (UNIMEP) & Educação Física & 4 & - \\
\hline & & Universidade São Judas Tadeu (USJT) & Educação Física & 4 & 4 \\
\hline & \multirow{3}{*}{ MG } & Universidade Federal de Minas Gerais (UFMG) & Ciências do Esporte & 4 & 4 \\
\hline & & Universidade Federal de Viçosa (UFV) & Educação Física & 3 & - \\
\hline & & $\begin{array}{l}\text { Universidade Federal do Triângulo Mineiro } \\
\text { (UFTM) }\end{array}$ & Educação Física & 3 & - \\
\hline & ES & Universidade Federal do Espírito Santo (UFES) & Educação Física & 3 & - \\
\hline \multirow{10}{*}{ SUL } & \multirow{5}{*}{ PR } & Universidade Federal do Paraná (UFPR) & Educação Física & 5 & 5 \\
\hline & & Universidade Estadual de Londrina (UEL) & \multirow{3}{*}{ Educação Física } & \multirow{3}{*}{4} & \multirow{3}{*}{4} \\
\hline & & & & & \\
\hline & & Universidade Estadual de Maringá (UEM) & & & \\
\hline & & Universidade do Norte do Paraná (UNOPAR) & Prescrição de Exercício Físico & 3 & - \\
\hline & \multirow[t]{2}{*}{ SC } & $\begin{array}{l}\text { Universidade do Estado de Santa Catarina } \\
\text { (UDESC) }\end{array}$ & $\begin{array}{l}\text { Ciências do Movimento } \\
\text { Humano }\end{array}$ & 3 & 3 \\
\hline & & Universidade Federal de Santa Catarina (UFSC) & Educação Física & 5 & 5 \\
\hline & \multirow{3}{*}{ RS } & $\begin{array}{l}\text { Universidade Federal do Rio Grande do Sul } \\
\text { (UFRGS) }\end{array}$ & $\begin{array}{l}\text { Ciências do Movimento } \\
\text { Humano }\end{array}$ & 5 & 5 \\
\hline & & Universidade Federal de Santa Maria (UFSM) & Educação Física & 3 & - \\
\hline & & Universidade Federal de Pelotas (UFPEL) & Educação Física & 3 & - \\
\hline \multirow{3}{*}{$\begin{array}{l}\text { CENTRO- } \\
\text { OESTE }\end{array}$} & \multirow{3}{*}{ DF } & Universidade de Brasília (UNB) & Educação Física & 3 & - \\
\hline & & Universidade Católica de Brasília (UCB) & Educação Física & 4 & 4 \\
\hline & & Universidade Federal do Mato Grosso (UFMT) & Educação Física & 3 & - \\
\hline \multirow{3}{*}{ NORDESTE } & $\mathrm{RN}$ & $\begin{array}{l}\text { Universidade Federal do Rio Grande do Norte } \\
\text { (UFRN) }\end{array}$ & Educação Física & 3 & - \\
\hline & SE & $\begin{array}{l}\text { Fundação Universidade Federal do Sergipe } \\
\text { (FUFSE) }\end{array}$ & Educação Física & 3 & - \\
\hline & $\mathrm{PE}$ & Universidade de Pernambuco (FESP/UPE) & Educação Física & 3 & - \\
\hline
\end{tabular}

Fonte: Adaptado da CAPES

No quadro 2 buscamos aprofundar a discussão a respeito da Pós-Graduação a nível regional, desse modo, destacamos os programas de Pós-Graduação strictu senso da região Sul do Brasil, enfatizando o cenário do Rio Grande do Sul (RS). O Curso de Mestrado em Ciências do Movimento Humano da UFRGS iniciou suas atividades em 1989. Entre seus objetivos estava a criação de um centro de produção de conhecimento e atividades em movimento humano, que capacitasse profissionais graduados a melhores níveis de desempenho no ensino, na pesquisa e na qualidade sociocultural. Dez anos após, o mesmo Programa, passa a oferecer 
um curso de Doutorado, consolidando-se como espaço de pesquisa e produção de conhecimento no $\mathrm{RS}^{8}$.

O mestrado em Educação Física da Universidade Federal de Pelotas (UFPEL) teve sua primeira turma de ingressantes em 2007. Mantendo a lógica de organização e a aproximação dos professores, o programa de mestrado, foi construído em torno das diferentes linhas de pesquisa, e de forma muito similar com outros programas dentro da área da Educação Física brasileira.

A Universidade Federal do Paraná (UFPR) teve seu curso de mestrado criado no ano de 2002, se consolidou com o passar dos anos, criando seu curso de doutorado, posteriormente no ano de 2007. Atualmente os programas de Pós-Graduação mantidos pela universidade possuem conceito 5, atribuído pela CAPES, contando com corpo docente de 19 professores.

Analisando a Universidade Estadual de Londrina (UEL), verificamos que esta, possui um dos cursos de mestrado mais recente da região Sul, criado em 2006, possuindo conceito 4 atribuído pela CAPES e conta com um corpo docente equivalente a outros programas tradicionais.

Os programas de Pós-Graduação em Educação Física do estado de Santa Catarina - UFSC e UDESC foram criados na década de 90, nos anos de 1996 e 1997, respectivamente. Após dez anos de consolidação os mesmos programas passam a oferecer um curso de doutorado, proporcionando maior à qualificação de seus programas.

A Universidade Federal de Santa Maria (UFSM), mesmo sendo uma das pioneiras nos investimentos da Pós-Graduação brasileira em Educação Física na década de 80 , por questões organizacionais e políticas passou por um período de reformulação, deixando de oferecer um curso de mestrado por um período de aproximadamente dez anos (2002-2012). Este período de amadurecimento dos docentes proporcionou uma nova reabertura do curso no ano de 2012, porém na época desta coleta de dados não havia nota de avaliação da CAPES, estando ainda na fase de estruturação.

Quadro 2 - Programas de Pós-Graduação na região sul do Brasil

\begin{tabular}{|c|c|c|c|c|c|}
\hline \multirow{2}{*}{ Instituição } & \multirow{2}{*}{ Nome do Programa } & \multicolumn{2}{|c|}{ Ano de Início } & \multirow{2}{*}{$\begin{array}{c}\text { Avaliação Trienal da } \\
\text { CAPES }\end{array}$} & \multirow{2}{*}{$\begin{array}{c}\text { Número de Docentes } \\
\text { Permanentes }\end{array}$} \\
\hline & & M & D & & \\
\hline UFRGS & Ciências do Movimento Humano & 1989 & 1999 & 5 & 17 \\
\hline UFPEL & Educação Física & 2007 & - & 3 & 16 \\
\hline UFPR & Educação Física & 2002 & 2007 & 5 & 19 \\
\hline UEL & Educação Física & 2006 & - & 4 & 19 \\
\hline UFSC & Educação Física & 1996 & 2006 & 5 & 18 \\
\hline UDESC & Ciências do Movimento Humano & 1997 & 2009 & 3 & 21 \\
\hline UFSM* & Educação Física & 2012 & - & - & - \\
\hline
\end{tabular}

Fonte: Adaptado da CAPES. * Sem avaliação Trienal pela CAPES.

Ao buscar mapear as estruturas de construção de cada um dos programas de Pós-Graduação e suas linhas de investigação científica, percebe-se que a grande problemática diz respeito aos docentes e suas áreas de conhecimento. $\mathrm{O}$ contexto profissional onde estão imersos, está pautado numa concepção de universidade atrelada às políticas avaliativas, que reconhece a pesquisa como avanço, mas ainda numa dimensão voltada para a produtividade, fazendo com que estes se sintam tensionados, externamente pelas exigências de produção acadêmica, e internamente pelos espaços a serem alcançados na própria instituição ${ }^{9-10}$. 
O Quadro 3, apresenta o cenário da Pós-Graduação na região Sul de acordo com as áreas de concentração e linhas de pesquisa, onde se percebe um volume maior de linhas de pesquisa relacionadas com a área das Ciências Biológicas/Saúde, e tal resultado vem ao encontro da discussão epistemológica no campo da produção de conhecimento na Educação Física. Ao visualizar as linhas apresentadas no quadro, percebe-se que das 28 linhas de pesquisa, 17 delas estão relacionadas às Ciências Biológicas/Saúde, e 11 relacionadas às Ciências Sociais e Humanas. Entretanto, não se pode afirmar categoricamente que uma linha pertence exclusivamente a uma área de conhecimento, uma vez que o campo da Educação Física, favorece a interface entre as linhas de pesquisa e a produção do conhecimento. Existe ainda, uma grande pluralidade epistemológica no que se refere aos conceitos e a terminologia empregada nas áreas de concentração e linhas de pesquisa, contribuindo para dificuldade no estabelecimento de fronteiras na área.

Quadro 3 - Área de concentração e linhas de pesquisa dos programas de Pós-Graduação na região sul do Brasil.

\begin{tabular}{|c|c|c|}
\hline Sigla & Área (s) de Concentração & Linhas de Pesquisa \\
\hline UFPR & - Exercício e Esporte. & $\begin{array}{l}\text { - Comportamento Motor; } \\
\text { - Atividade Física e Saúde; } \\
\text { - Fisiologia da Performance; } \\
\text { - Sociologia para o Esporte e o Lazer. }\end{array}$ \\
\hline UFRGS & $\begin{array}{l}\text { - Movimento Humano, Cultura e } \\
\text { Educação; } \\
\text { - Movimento Humano, Saúde e } \\
\text { Performance. }\end{array}$ & $\begin{array}{l}\text { - Representações Sociais do Movimento Humano; } \\
\text { - Formação de Professores e Prática Pedagógica; } \\
\text { - Atividade Física e Saúde; } \\
\text { - Atividade Física e Performance; } \\
\text { - Neuromecânica do Movimento Humano; } \\
\text { - Desenvolvimento da Coordenação e do Controle Motor. }\end{array}$ \\
\hline UFSC & $\begin{array}{l}\text { - Atividade Física Relacionada à Saúde; } \\
\text { - Cineantropometria e Desempenho } \\
\text { Humano; } \\
\text { - Teoria e Prática Pedagógica em } \\
\text { Educação Física. }\end{array}$ & $\begin{array}{l}\text { - Processos e Programas de Promoção da Atividade Física; } \\
\text { - Educação Física, Condições de Vida e Saúde; } \\
\text { - Teorias sobre o Corpo e Movimento Humano na Sociedade; } \\
\text { - Teorias Pedagógicas e Didáticas do Ensino da Educação Física; } \\
\text { - Esporte e Lazer em Culturas Contemporâneas; } \\
\text { - Estudo da Inter-relação Morfologia e Função; } \\
\text { - Interação Exercício Físico, Aptidão Física e Desempenho no Esporte e no } \\
\text { Trabalho. }\end{array}$ \\
\hline UEL - UEM & - Estudos do Movimento Humano. & $\begin{array}{l}\text { - Aquisição e Desempenho de Habilidades Motoras; } \\
\text { - Aspectos Fisiológicos do Exercício Físico; } \\
\text { - Atividade Física Relacionada à Saúde; } \\
\text { - Formação e Intervenção em Educação Física. }\end{array}$ \\
\hline UDESC & $\begin{array}{l}\text { - Estudos Biocomportamentais do } \\
\text { Movimento Humano. }\end{array}$ & $\begin{array}{l}\text { - Biomecânica; } \\
\text { - Desenvolvimento Humano; } \\
\text { - Atividade Física e Saúde. }\end{array}$ \\
\hline UFPEL & - Atividade Física, Esporte e Escola. & $\begin{array}{l}\text { - Atividade Física, Escola e Sociedade; } \\
\text { - Atividade Física, Nutrição e Saúde; } \\
\text { - Memória, Corpo, Esporte e Formação Profissional; } \\
\text { - Aprendizagem, Desenvolvimento, formação e Currículo. }\end{array}$ \\
\hline UFSM & - Educação Física, Saúde e Sociedade. & $\begin{array}{l}\text { - Aspectos Socioculturais e Pedagógicos da Educação Física; } \\
\text { - Aspectos Biológicos e Comportamentais da Educação Física e da Saúde. }\end{array}$ \\
\hline
\end{tabular}

Fonte: Adaptado da CAPES.

\section{DISCUSSÃO}

A partir das mudanças ocorridas no cenário da Pós-Graduação brasileira como qualificação docente, consolidação das linhas de pesquisa, incremento e diversidade da produção intelectual, aumento das discussões (tanto internas quanto ex- 
ternas aos Programas), houve um crescimento da área da Educação Física. Hoje é possível mapear cada instituição, linhas de pesquisa, suas áreas de interesse e a concepção de ciência que norteia a formação dos futuros mestres e doutores ${ }^{11-12}$.

Conforme os resultados apresentados no Quadro 1, podemos considerar que a mobilização em torno de melhor reconhecimento de cada programa vem ao encontro das políticas avaliativas governamentais, fazendo com que cada Programa de Pós-Graduação busque estar nos níveis desejados a fim de manterem-se em condições de receber financiamento para as pesquisas; captação de cotas de bolsas; padrões consideráveis nas avaliações da CAPES ou outras agências financiadoras.

Com os investimentos na qualificação docente e com a consolidação da pesquisa, a Pós-Graduação passa a ser um lócus de produção do conhecimento e para tal, ela mergulha em uma ideia da busca por competência e reconhecimento dos pares. Para Afonso ${ }^{8}$, os espaços de produção de conhecimento que foram sendo consolidados, bem como a criação dos programas de Pós-Graduação em Educação Física não se devem ao acaso. Eles se inserem no bojo das orientações contidas no conjunto orgânico dos documentos constitutivos da Política Brasileira de Pós-Graduação.

Considerando os resultados referentes à configuração dos programas de PósGraduação e sua avaliação trienal pela CAPES, a área da Educação Física tem buscado melhores índices avaliativos, que se relacionam diretamente com a produção docente e agregam ainda as condições de desenvolvimento da pesquisa no âmbito de cada programa.

Segundo Chau ${ }^{10}$, as universidades vivem pressionadas e pressionam seus docentes, para atingirem melhores índices de produção acadêmica, de maneira que estes possam produzir para competir com outras instituições que compartilham da mesma demanda de resultados. A autora ainda explica que, o contexto profissional onde estão imersos, está pautado numa concepção de universidade atrelada às políticas avaliativas, que reconhece a pesquisa como avanço, mas ainda numa dimensão muito voltada para a produtividade, fazendo com que estes se sintam tensionados, externamente pelas exigências de produção acadêmica, e internamente pelos espaços a serem alcançados na própria instituição.

Os resultados apresentados no Quadro 2, refletem a realidade dos programas de Pós-Graduação na região Sul, segundo a avaliação da CAPES e configuração do quadro docente. Nos últimos anos o compromisso com a pesquisa, tendo por base a qualificação docente, no interior das IES, alcançou um patamar diferenciado, tanto no processo de formação inicial quanto na Pós-Graduação. A pluralidade de formação dos docentes tem contribuído para que os programas obtenham melhores resultados nas avaliações institucionais, contribuindo para a sua consolidação no cenário da pesquisa na área da Educação Física.

$\mathrm{O}$ atual sistema de avaliação da CAPES é regido por indicadores, que atribuem aos cursos um conceito que varia de 1-7 pontos dos quais a produção científica tem sido destacada como a principal diferenciadora entre os programas ${ }^{11}$.

No presente estudo, os resultados sinalizaram uma maior consolidação da área biológica/saúde (Quadro 3), visto que existe uma maior concentração de linhas vinculadas a ela, resultado dos investimentos em qualificação docente e infraestrutura institucional. Segundo Cunha ${ }^{13}$, ainda podemos considerar que "a universidade é uma instituição que organiza e reflete os campos científicos”. Neste sentido há perfis diferentes dos diversos grupos de carreiras, já que os valores, em cada campo científico, podem variar, e o que cada um deles é percebido como interessante é o que tem chance de ser reconhecido como importantes para os outros, tanto para os pares de mesma área ou campo, como para a comunidade científica mais ampla ${ }^{10}$. 
Segundo estudo realizado por Rigo et al. ${ }^{11}$ existe uma reconfiguração do quadro docente dentro dos programas, onde 142 docentes estavam inseridos na área das Ciências Sociais e Humanas e 260 nas áreas das Ciências Biológicas e da Saúde. Os autores argumentam que, a formação de grupos de pesquisa e a produção coletiva, refletem as diferenças entre as áreas de concentração e linhas de pesquisa, onde aquelas com um menor destaque enfrentam maiores dificuldades do que aquelas já consolidadas. Tentando explicar este fenômeno, os autores argumentam que em função das singularidades epistemológicas das Ciências Sociais e Humanas a internacionalização da produção do conhecimento possui um valor distinto daquele que vigora nas Ciências Biológicas e da Saúde.

Cunha ${ }^{15}$ argumenta que a comunidade científica não é um espaço neutro, necessariamente só regido por idealismos. Ela reflete as mesmas disputas de qualquer campo social na organização capitalista da sociedade. Para Ortiz ${ }^{14}$, seguindo as ideias de Bourdieu, em um determinado campo, os investimentos dos pesquisadores dependem tanto da importância, quanto da sua natureza, e da importância de seu capital atual e potencial de reconhecimento, bem como de sua posição atual e potencial no campo. São essas correlações que determinam as propensões de investimento do capital científico.

As transformações vividas na área, seu amadurecimento, sua preocupação com a qualificação profissional, sua expressão científica em espaços legitimados cientificamente, conquistas no que diz respeito à regulamentação profissional, bem como a consolidação dos programas de Pós-Graduação ocorridos recentemente, também são geradas a partir das demandas em atender os critérios de produção docente e melhor definição das linhas de investigação e pesquisa. O que se percebe é um relativo aumento do número de programas, com perspectivas de impactar a área oportunizando traçar melhores condições para se pensar a uma formação plural e diferenciada no interior das universidades.

O mapeamento realizado acena com alguns elementos para se pensar que exista uma tendência para a pluralidade das áreas dentro dos programas, onde a análise biológica do movimento humano, com reconhecimento nacional e internacional através das pesquisas produzidas, seja mais enfatizada.

Nos últimos anos é visível o avanço na estruturação e organização da Pós-Graduação brasileira, tanto no que se refere ao número de programas, bem como ao nível de qualificação dos professores e das pesquisas nas mais diferentes áreas de conhecimento. O sistema de avaliação da CAPES tem colaborado para a maior visibilidade dos programas e sinalizado como devem ser conduzidas as reformulações de maneira que seja garantidas a qualidade e expansão de novos cursos.

Há um momento de favorecimento para a ampliação das estratégias de consolidação dos programas de Pós-Graduação, no interior da área da Educação Física, na região sul. Isto tem acontecido pela necessidade de formação profissional, pelo incentivo à qualificação dos docentes, pela ampliação do número de vagas, bem como pela demanda do mercado de trabalho. Ao finalizar este trabalho, a partir dos dados levantados foi possível perceber que existe um esforço e uma vontade política coletiva para que se construam diferentes espaços/lócus de produção do conhecimento com capacidade de competir com outros centros de excelência em ensino e de pesquisa já consolidados no Brasil.

Ao mesmo tempo com base neste cenário descrito, consideramos que o momento vivido no campo acadêmico-científico, a partir dos indicativos de qualidade impostos pelas agências de fomentos de pesquisa e pelos índices a serem atingidos, tem gerado tensões tanto no que diz respeito ao trabalho docente quanto às 
áreas de produção do conhecimento. Por outro lado, é possível que a partir de um reordenamento e aproximação das áreas e linhas de pesquisa, possa haver uma interface favorecendo a produção do conhecimento, o que contribui para o desenvolvimento da Pós-Graduação em Educação Física.

\section{Contribuição dos autores}

MR Afonso e HM Quadros idealizaram o artigo. JAB Ribeiro gerenciou a coleta de dados junto aos sítios das instituições e organizou as tabelas/figuras. Todos os autores participaram da redação/revisão do manuscrito.

\section{REFERÊNCIAS}

1. Afonso MR, Ramos MGG, Garcia TEM. Movimentos de Expansão no Ensino Superior na Universidade Brasileira. In: XXX International Congress of the Latin American Studies Association - LASA. Anais... São Francisco, Califórnia, 2012.

2. Franco, MEP. Inovação e Pesquisa na Universidade: racionalidade e produção individual/ cooperada. Relatório de Pesquisa. Universidade Federal do Rio Grande do Sul. Grupo de Estudos sobre a Universidade. Porto Alegre: UFRGS, 1996.

3. Brasil. IV Plano Nacional de Pós-Graduação - 2005/2010. Coordenação de Aperfeiçoamento de Pessoal de Nível Superior. Ministério da Educação (MEC). Disponível em: http://www. capes.gov.br/images/stories/download/editais/PNPG_2005_2010.pdf. Acesso em: 10 ago. 2010.

4. Kokubun E. Pós-Graduação em Educação Física. Revista Brasileira de Educação Física e Esporte. São Paulo, v.20, p.31-32, set. 2006.

5. Kokubun E. Pós-Graduação em Educação física no Brasil: indicadores, objetivos dos desafios e das perspectivas. Revista Brasileira de Ciências do Esporte. Campinas, v.24, n. 2, p.9-26, jan. 2003.

6. Molina V. Pós-Graduação em Educação Física: um olhar sobre o Programa da ESEF/UFRGS. Revista Brasileira de Ciências do Esporte, n.20, set. 2008.

7. Cellard A. A análise documental. In: POUPART, J. et al. A pesquisa qualitativa: enfoques epistemológicos e metodológicos. Petrópolis, Vozes, 2008.

8. Afonso MR. A articulação do conhecimento pós-graduação/graduação e os possíveis nexos com a formação profissional em Educação Física. In: KRUG, Hugo Norberto; PEREIRA, Flávio Medeiros; AFONSO, Mariângela da Rosa (Orgs.). Educação Física: formação e práticas pedagógicas. Pelotas: Editora e Gráfica Universitária, 2009. 234p.

9. Sguissardi V. Avaliação universitária em questão: reformas do Estado e da Educação Superior. Campinas, SP: Autores Associados, 1997.

10. Chauí, MS. Escritos sobre a universidade. São Paulo, SP: Editora UNESP, 2001.

11. Rigo LC, Ribeiro GM, Hallal PC. Unidade na diversidade: desafios para a Educação Física no século XXI. Revista Brasileira de Atividade Física e Saúde. Vol. 16, n. 11, p. 339-345. 2011.

12. Quadros, HM. Mestrados na área de educação física no estado do Rio Grande do Sul: um estudo sobre o perfil dos egressos [dissertação de mestrado]. Pelotas: Universidade Federal de Pelotas, 2012.

13. Cunha MI. Docência na universidade, cultura e avaliação institucional: saberes silenciados em questão. Revista Brasileira de Educação. Vol.11, n. 32, mai/ago. 2006.

14. Ortiz R. 1983. Bourdieu - Sociologia. São Paulo: Ática. Coleção Grandes Cientistas Sociais, vol. 39. p. 122-155.

15. Cunha MI. Aula Universitária: Inovação e Pesquisa. In: LEITE, Denise; MOROSINI, Marilia. Universidade Futurante. Campinas: Papirus, 1997.

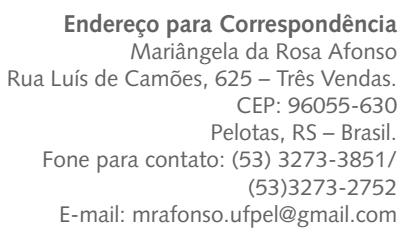

Recebido 29/10/2013

Revisado 05/11/2013

Aprovado $07 / 11 / 2013$ 